\title{
Genome-wide analyses of HTLV-1aD strains from Cape Verde, Africa
}

\author{
Louise Zanella1, Isabel de Pina-Araujo I², Mariza G Morgado ${ }^{3}$, Ana Carolina Vicente ${ }^{1 /+}$ \\ 'Fundação Oswaldo Cruz, Instituto Oswaldo Cruz, Laboratório de Genética Molecular de Microrganismos, Rio de Janeiro, RJ, Brasil \\ ${ }^{2}$ Universidade de Cabo Verde, Departamento de Ciência e Tecnologia, Praia, Santiago, Cabo Verde \\ ${ }^{3}$ Fundação Oswaldo Cruz, Instituto Oswaldo Cruz, Laboratório de AIDS e Imunologia Molecular, Rio de Janeiro, RJ, Brasil
}

We characterised and reported the first full-length genomes of Human T-cell Lymphotropic Virus Type 1 subgroup HTLV-1aD (CV21 and CV79). This subgroup is one of the major determinants of HTLV-1 infections in North and West Africa, and recombinant strains involving this subgroup have been recently demonstrated. The CV21 and CV79 strains from Cape Verde/Africa were characterised as pure HTLV-1aD genomes, comparative analyses including HTLV-1 subtypes and subgroups revealed HTLV-1aD signatures in the envelope, pol, and $p X$ regions. These genomes provide original information that will contribute to further studies on HTLV-la epidemiology and evolution.

Key words: HTLV-1, HTLV-1aD complete genome - HTLV-1aD signatures - Cape Verde - Africa - GC Content

The Human T-cell Lymphotropic Virus Type 1 (HTLV1) belongs to the Retroviridae family in the Deltaretrovirus genus and is the first oncogenic human retrovirus discovered (Gallo 2005). HTLV-1 is associated with severe diseases, such Adult T-cell Leukemia, Tropical Spastic Paraparesis and other inflammatory diseases that are endemic in regions such as Southwestern Japan, the Caribbean, Sub-Saharan Africa, and South America, including Brazil (Verdonck et al. 2007). A recent study on the worldwide prevalence of HTLV-1 estimated between 5-10 millions infected individuals, most of them in Central/ South America and Africa (Gessain \& Cassar 2012). Its genome encodes the typical retrovirus proteins (Gag, Pol and Env), HTLV-1-specific regulatory proteins (Tax and Rex) and the long terminal repeat (LTR) (Lairmore et al. 2011). Phylogenetic analyses based on the LTR region segregated HTLV-1 into seven major subtypes (a-g): cosmopolitan subtype (1a), African subtypes (1b, 1d, le, 1f and lg) and Australo-Melanesian subtype (1c) (Vandamme et al. 1994). The cosmopolitan subtype (1a) is widespread worldwide and classified into five subgroups: (A) Transcontinental, (B) Japanese, (C) West African, (D) North African, and (E) Peruvian Black. The genetic diversity in HTLV is driven by the error-prone reverse transcriptase. However, this diversity has also been demonstrated with the recent identification of HTLV-1 West African strains resulting from recombination involving the LTR region from the HTLV$1 \mathrm{aC}$ and HTLV-laD subgroups (Desrames et al. 2014).

Most genetic and evolutionary studies on HTLV-1 were based on partial genome sequences, particularly the LTR region. However, complete genome sequences of the most prevalent HTLV-1a subgroups (except HTLV1aD) are currently available (Pessôa et al. 2014).

doi: 10.1590/0074-02760160227

Financial support: CNPq and CAPES (scholarships).

+ Corresponding author: anapaulo@ioc.fiocruz.br

Received 25 May 2016

Accepted 29 June 2016
The phylogenetic analysis, based on the LTR region, revealed the presence of the HTLV-1aD subgroup in Cape Verde Archipelago in Africa (personal communication).

In this study, we present the complete genome sequence of two HTLV-1aD strains from Cape Verde along with genome-wide comparative analyses including HTLV-la prevalent subgroups.

The genome of the CV21 and CV79 strains from Cape Verde were amplified through nested polymerase chain reaction (PCR) using degenerate HTLV primers and the PCR-based genome-walking strategy (Supplementary Table I). Amplicons were sequenced in the Sanger Platform at Fiocruz using the BigDye Terminator v3.1 Cycle sequencing kit (Applied Biosystems, Foster City, CA, EUA). Genome sequences were deposited in the GenBank under the accession numbers: KX430030and KX430031.

The CV 21 and CV79 genomes have a total length of 9,037 bp from the 5' to $3^{\prime}$ provirus LTRs. All HTLV1 characteristic genomic regions were present and syntenic in these two HTLV-laD when compared with the other HTLV-1 (Fig. 1). Their global GC content were $53 \%$, consistent with the GC-rich characteristic of HTLV-1 (Zanella et al. 2012). Nevertheless, a wide range of GC contents, from $49 \%$ (protease) to $61 \%$ (p12), were observed between different coding regions as $51 \%$ (integrase and envelope), 52\% (reverse transcriptase), 53\% (tax), 55\% (HBZ), 56\% (gag), 57\% (p30), and 58\% (rex).

The phylogenetic analysis based on the LTR region confirmed that CV21 and CV79 belong to the HTLV-1aD subgroup, which is spread in North African countries such as Senegal and Guinea-Bissau (Zehender et al. 2008).

The phylogenomic analysis comparing the HTLV$1 \mathrm{aD}$ and HTLV-1 subgroups ( $\mathrm{aA}, \mathrm{aB}$, and $\mathrm{aC}$ ) and subtypes (b and c) (Fig. 2) showed that the HTLV-laD genome shares $98 \%$ identity with HTLV-1a subgroups (aA, $\mathrm{aB}$, and $\mathrm{aC}$ ) while its identity with the $\mathrm{b}$ and $\mathrm{c}$ subtypes are $96 \%$ and $91 \%$, respectively. The presence of possible recombination on CV21 and CV79 strains was evaluated by phylogenetic analysis in the LTR and env HTLV-1aD regions. This cluster formed showed no evidence of recombination within these two HTLV-laD genomes. 


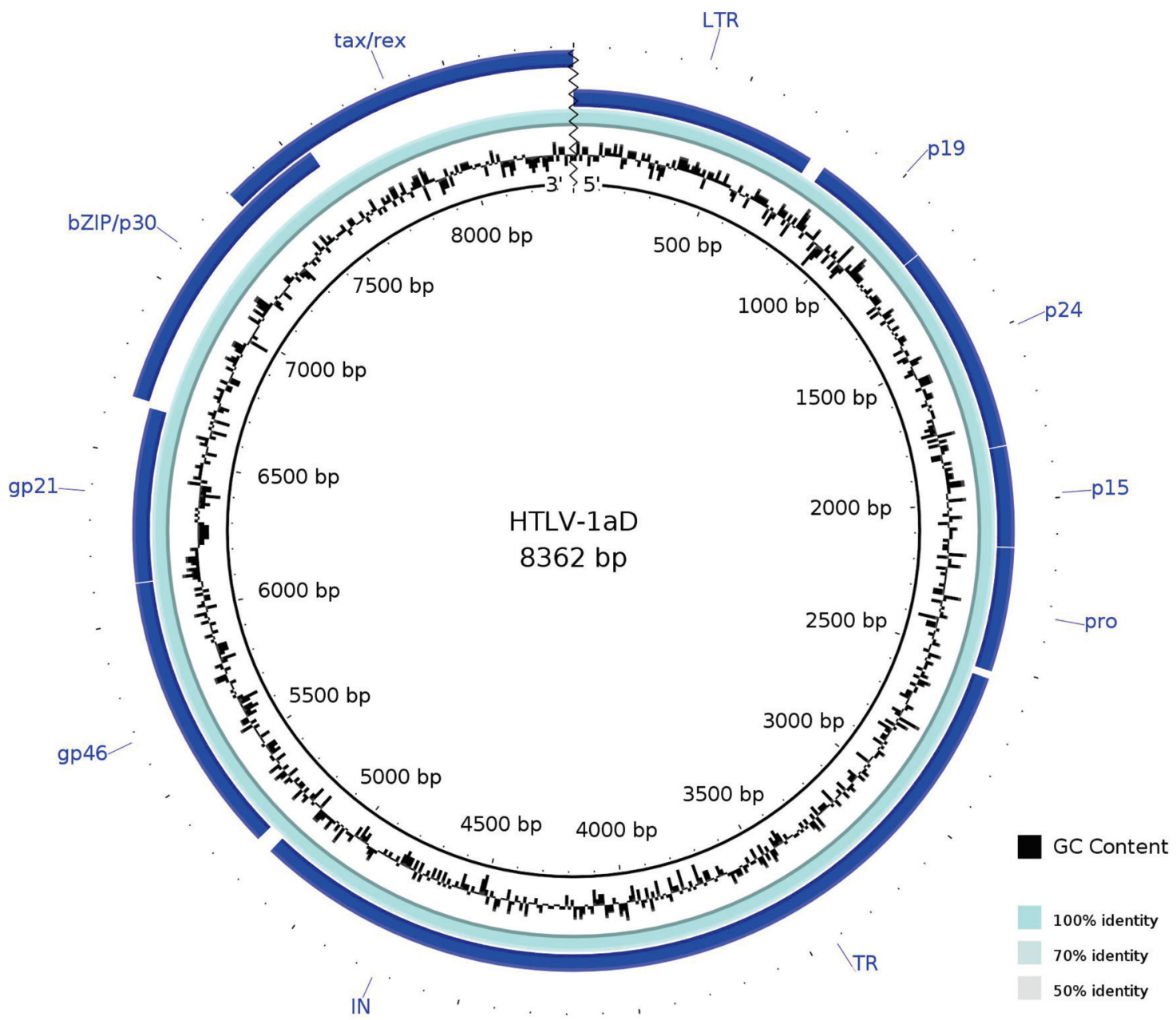

Fig. 1: genome maps and features of HTLV-1aD subgroup considering only LTR5'. The inner circle represents the HTLV-1aD genome following by percent of GC content and the other circles represents the genome annotation.



Fig. 2: Maximum Likelihood tree based on HTLV-1 entire genome sequences. Sequences derived from this study are in red. Numbers besides internal branches indicate bootstrap values based on 1000 replicates. 
HTLV-laD is characterised by unique amino acids residues, when compared with other subgroups and subtypes which share the same conserved residue in the following positions: gp46 env A59V; gp21 env L454F; TR K486R; p30 S105N; and HBZ isoforms M/I and V/A. Regardless of presenting unique residues, the charges and polarity properties of these residues were maintained in HTLV-1aD. In some other positions, HTLV-laD present specific amino acid (aa) residues, which is contrasting to what is observed in other subgroups and subtypes that do not share the same conserved residues in the following positions: TR V/A310I; p12 D/N/E/A26K; HBZ SP2 isoform L/P13R. Moreover, HTLV-1aD specific substitutions in p12 and HBZ showed different charge and polarity aa properties (Supplementary Tables II-III).

The two HTLV-1aD complete genomes presented in this study represent original information about a subgroup that is prevalent in African countries. These genomes provide information that will contribute to further studies on HTLV-la epidemiology and evolution.

\section{ACKNOWLEDGEMENTS}

To the assistance from the PDTIS/Fiocruz sequencing platform.

\section{REFERENCES}

Calattini S, Betsem E, Bassot S, Chevalier SA, Mahieux R, Froment A, et al. New strain of human T lymphotropic virus (HTLV) type 3 in a Pygmy from Cameroon with peculiar HTLV serologic results. J Infect Dis. 2009; 199(4): 561-4.

Desrames A, Cassar O, Gout O, Hermine O, Taylor GP, Afonso PV, et al. Northern African strains of human T-lymphotropic virus type 1 arose from a recombination event. J Virol. 2014; 88(17): 9782-8.

Furukawa Y, Usuku K, Izumo S, Osame M. Human T cell lymphotropic virus type I (HTLV-I) p12I is dispensable for HTLV-I transmission and maintenance of infection in vivo. AIDS Res Hum Retroviruses. 2004; 20(10): 1092-9.

Gallo RC. History of the discoveries of the first human retroviruses: HTLV-1 and HTLV-2. Oncogene. 2005; 24(39): 5926-30.
Gessain A, Cassar O. Epidemiological aspects and world distribution of HTLV-1 infection. Front Microbiol. 2012; 3: 388.

Lairmore MD, Anupam R, Bowden N, Haines R, Haynes RA 2nd, Ratner L, et al. Molecular determinants of human T-lymphotropic virus type 1 transmission and spread. Viruses. 2011; 3(7): 1131-65.

Liu HF, Vandamme AM, Kazadi K, Carton H, Desmyter J, Goubau P. Familial transmission and minimal sequence variability of human T-lymphotropic virus type I (HTLV-I) in Zaire. AIDS Res Hum Retroviruses. 1994; 10(9): 1135-42.

Pessôa R, Watanabe JT, Nukui Y, Pereira J, Kasseb J, de Oliveira ACP, et al. Molecular characterization of human T-cell lymphotropic virus type 1 full and partial genomes by illumina massively parallel sequencing technology. PLoS ONE. 2014; 9(3): e93374.

Vandamme AM, Liu HF, Goubau P, Desmyter J. Primate T-lymphotropic virus type I LTR sequence variation and its phylogenetic analysis: compatibility with an African origin of PTLV-I. Virology. 1994; 202(1): 212-23.

Vandamme AM, Van Laethem K, Liu HF, Van Brussel M, Delaporte $\mathrm{E}$, Costa CMC, et al. Use of a generic polymerase chain reaction assay detecting human T-lymphotropic virus (HTLV) types I, II and divergent simian strains in the evaluation of individuals with indeterminate HTLV serology. J Med Virol. 1997; 52(1): 1-7.

Verdonck K, González E, Van Dooren S, Vandamme A, Vanham G, Gotuzzo E. Human T-lymphotropic virus 1: recent knowledge about an ancient infection. Lancet Infect Dis. 2007; 7(4): 266-81.

Yang YC, Hsu TY, Liu MY, Lin MT, Chen JY, Yang CS. Molecular subtyping of human T-lymphotropic virus type I (HTLV-I) by a nested polymerase chain reaction-restriction fragment length polymorphism analysis of the envelope gene: two distinct lineages of HTLV-I in Taiwan. J Med Virol. 1997; 51(1): 25-31.

Zanella L, Otsuki K, Marin MA, Bendet I, Vicente AC. Complete genome sequence of Central Africa human T-cell lymphotropic virus subtype 1b. J Virol. 2012; 86(22): 12451.

Zehender G, Ebranati E, de Maddalena C, Gianelli E, Riva A, Rusconi S, et al. Description of a "trans-Saharan" strain of human T-lymphotropic virus type 1 in West Africa. J Acquir Immune Defic Syndr. 2008; 47(3): 269-73. 